\title{
Effect of Total Versus Partial Assisted Hatching on the Clinical Pregnancy Rates in Assisted Reproduction Technology
}

\author{
Bulent Emre BILGIC ${ }^{1}$, Enis OZKAYA², Cigdem YAYLA ABIDE ${ }^{2}$, Semra KAYATAS ESER ${ }^{2}$, \\ Ilhan SANVERDI', Tayfun KUTLU²
}

Istanbul, Turkey

\begin{abstract}
OBJECTIVE: The objective of this study was to investigate the effect of total or partial assisted hatching on the clinical pregnancy rates in assisted reproduction technology.

STUDY DESIGN: This was a case-control study conducted from the beginning of January 2016 to the end of June 2017. A total of 404 cycles were included in this case-control study. Study population was divided into 3 groups: Group 1: Partial assisted hatching $(n=118)$, Group 2: Total assisted hatching $(n=81)$ and Control group $(n=205)$.

RESULTS: In women of all ages, clinical pregnancy rates were similar between groups with total or partial assisted hatching compared to control group $(p>0.05)$. The rates were also similar in subgroups of women with blastocyst or cleavage stage embryo transfers $(p>0.05)$. Partial or total embryo hatching did not result in favorable outcome compared to the control group either in women over 35 or younger than 35 years of age $(p>0.05)$. In whole study group, pregnancy rate was significantly higher in the group with blastocyst stage embryo transfers $(22.4 \%$ versus $48.8 \%, p<0.05)$.
\end{abstract}

CONCLUSION: Partial or total assisted hatching do not have any impact on the clinical pregnancy rates, no significant impact was determined in the subgroup of women either.

Keywords: Assisted hatching, Intracytoplasmic sperm injection, Implantation, In vitro fertilization, Partial and total assisted hatching

Gynecol Obstet Reprod Med 2019;25(1):22-27

\section{Introduction}

A successful hatching of an embryo from its zona pellucida (ZP) has significant impact on the assisted reproduction

\footnotetext{
${ }^{1}$ Department of Embryology, ${ }^{2}$ Department of Reproductive Medicine and Infertility Zeynep Kamil Women and Children's Health Training and Research Hospital, Istanbul Turkey

Address of Correspondence: Cigdem Yayla Abide Zeynep Kamil Women and Children's Health Training and Research Hospital, Department of Obstetrics and Gynecology 34668 Uskudar, Istanbul Turkey cigdemabide@gmail.com

Submitted for Publication: $\quad 20.02 .2018$ Accepted for Publication: $\quad 12.04 .2018$
}

ORCID IDs of the authors:

BE.B.: 0000-0002-7298-8949, E.O: 0000-0001-6580-1237,

C.YA.: 0000-0001-5437-7987, S.KE.: 0000-0003-4675-808X, I.S: 0000-0001-9174-6681, T.K.: 0000-0001-9855-0331

\begin{tabular}{|c|c|}
\hline Quick Response Code: & Access this article online \\
\cline { 2 - 2 } & Website: www.gorm.com.tr \\
& e- mail: info@gorm.com.tr \\
\cline { 2 - 2 } & DOI:10.201613/GORM.2018.780 \\
\hline
\end{tabular}

How to cite this article: Bilgic BE. Ozkaya E. Yayla Abide C. Kayatas Eser S. Sanverdi I. Kutlu T. Effect of Total Versus Partial Assisted Hatching on the Clinical Pregnancy Rates in Assisted Reproduction Technology. Gynecol Obstet Reprod Med 2019;25(1):22-27 technology (ART) outcome (1-3). It was proposed that the elasticity of the ZP might be lost secondary to the long-term exposure of embryos to the in-vitro culture (4). Following the first report of pregnancy after assisted hatching $(\mathrm{AH})$, several approaches have been introduced to improve pregnancy rates in ART (5-7). With the pool of evidence, the restricted group of cases were proposed to have a favorable outcome with the $\mathrm{AH}$ (8-10). As a consequence, two meta-analyses demonstrated that $\mathrm{AH}$ has a positive impact on cycle outcome for the patients with repeated implantation failures $(11,12)$. Some different techniques for $\mathrm{AH}$ have been introduced up to date including chemical, mechanical, enzymatic, piezo-micromanipulation or laser. As a result, the technique with laser was shown to have some advantages over chemical or mechanical drilling procedures (13-18).

In addition to the best technique, the effect of the size of $\mathrm{AH}$ was also questioned in several studies, a partial hatching was shown to result in a higher implantation rate (19). Another study showed a higher implantation rate in the group with blastocyst stage embryo transfer and partial assisted hatching in this group resulted in a favorable outcome (20). However, there is still no clear evidence indicating the role of $\mathrm{AH}$ on the outcome of cases without known poor prognosis with their first fresh cycles (21). 
The objective of this study was to investigate the effect of total versus partial assisted hatching on the clinical pregnancy rates in ART in cases without known poor prognosis with their first fresh cycles.

\section{Material and Method}

The prospective comparative non-randomized study was conducted in the Zeynep Kamil Maternity and Children Education and Research Hospital Reproductive Endocrinology and ART department Istanbul-Turkey. Permission was obtained from the Institutional Ethics Committee for the conduct of this study. Written informed consent was obtained from all study participants. The study period was between the beginning of January 2016 to the end of June 2017.

A total of 404 cycles were included in the present study. ICSI patients assigned to one of the 3 groups: Group 1: Cycles with partial AH ( $\mathrm{n}=118)$, Group 2: Cycles with total $\mathrm{AH}(\mathrm{n}=81)$ and control group consisted of cycles without AH $(n=205)$.

\section{Participants}

In all patients, artificial reproductive techniques were indicated for unexplained infertility. Unexplained infertility was diagnosed when a patient was infertile with normal ovulatory and tubal functions, and her partner had a normal sperm count parameters (22). These were determined by menstrual cycle regularity, hysterosalpingography and semen analysis, respectively.

Exclusion criteria include; cycles without grade 1 embryos, frozen embryo or donor embryo transfers.

\section{Ovarian stimulation protocols}

For all participants with the first cycle without known poor prognosis, a gonadotropin-releasing hormone antagonist protocol was used for in vitro fertilization/intracytoplasmic sperm injection (IVF/ICSI) (23). On the second day of the menstrual cycle, 150-300 IU of recombinant follicle-stimulating hormone (r-FSH) (Gonal-FTM, Merck-Serono, Geneva, Switzerland) were administered, and mean follicular growth was monitored via two-dimensional transvaginal sonography (TVS). The daily dosage of r-FSH was adjusted from day 5 of stimulation according to ovarian response. The antagonist (Cetrorelix $^{\mathrm{TM}}$, Merck-Serono, Geneva, Switzerland) was administered at a dose of $0.25 \mathrm{mg} /$ day when follicular size reached $12 \mathrm{~mm}$ (24). When follicular size reached $18 \mathrm{~mm}$, $250 \mu \mathrm{cg}$ recombinant human chorionic gonadotropin (hCG) (Ovitrelle $^{\mathrm{TM}} 250 \mu \mathrm{g} / 0.5 \mathrm{~mL}$ Merck-Serono, Geneva, Switzerland) was administered subcutaneously, and the ovum pick was performed 34-36 hours afterward. ICSI was applied for each oocyte obtained by follicular puncture. Subsequently $8 \%$ vaginal progesterone gel (Crinone $^{\mathrm{TM}}$ Merck-Serono, Geneva, Switzerland) was applied twice daily, and continued until 10 weeks in patients who became pregnant.

Laser assisted hatching technique

Assisted hatching was performed $1 \mathrm{~h}$ before embryo trans- fer for both total and partial zona dissection. Procedure was applied under an Olympus inverted microscope IX-70 $(\times 4,10$, 20 , and 40 objectives) by Fertilase Microsurgical Laser Unit (MMT Medical Technologies) and a display monitor (MMT Medical Technologies, Montreux SA, Switzerland) (25). Type of $\mathrm{AH}$ was determined in non-randomized manner by the embryologist blinded for the patient characteristics, stimulation outcome, and number of oocytes retrieved.

\section{Embryo Transfer Protocol}

Embryo transfer (ET) was performed on day 3 under ultrasound guidance using a Wallace catheter (Marlow/Cooper Surgical, Shelton, CT, USA). After the visualization of the cervix at the dorsal lithotomy position, and the removal of cervical mucus gently with an aspiration catheter, the ET was performed under the guidance of transabdominal sonography (Logic 200.5 MHz transabdominal probe, General Electric, USA) with the partly filled bladder using a soft transfer catheter (Prodimed ZI, France), In all cases, an elective single grade 1 cleavage or blastocyst stage embryo was transferred.

\section{Clinical Outcomes}

The serum levels of beta-subunit of hCG ( $\beta$-hCG) were measured after two weeks. If they were more than or equal to normal levels $(5 \mathrm{mIU} / \mathrm{mL})$, the patient was considered to have a successful implantation. The primary outcome measure was the clinical pregnancy rate. All clinical pregnancies (i.e., those with an intrauterine sac) were defined by ultrasound visualization: $\mathrm{Sac}=$ intrauterine sac at $\geq 5$ weeks, Fetus $7+=$ viable fetus at $\geq 7$ weeks with the positive fetal heartbeat.

\section{Statistical analysis}

Analyses were carried out by SPSS version 15 . Continuous variables were not normally distributed and were given as median (range), unless indicated. Statistical comparison was carried out by Student-t, Mann-Whitney, and chisquare tests. Multivariate regression analysis was used to determine adjusted associations. Where appropriate, a $p$-value (two-tailed) of $<0.05$ was considered significant.

Subgroup analyses was undertaken to compare the two $\mathrm{AH}$ techniques in women with blastocyst or cleavage stage embryo transfers, and cases $<35$ years or older than 35 years of age.

\section{Results}

Table 1 summarizes the demographic, and clinical characteristics of the whole study population who underwent 404 cycles with an indication of unexplained infertility.

There were significant differences between groups with cleavage versus blastocyst stage embryo transfers in terms of age, number of total, and metaphase II oocytes $(p<0.05$, table 2).

There were significant differences regarding the age, serum FSH, total gonadotropin dose, number of total and 
metaphase II oocytes among groups with partial, total or no AH $(p<0.05$, table 3$)$. Pregnancy rates were similar among partial, total or no AH in the whole study population $(25.4 \%$, $25.9 \%, 24.9 \%$; respectively, $p>0.05$ ).

Pregnancy rates were similar among groups with partial, total and no assisted hatching in subgroup of cases with cleavage stage embryo transfers $(25.4 \%, 19.7 \%, 21.5 \%$; respectively, $p>0.05$ ). Partial assisted hatching was associated with the lowest pregnancy rate in the group with blastocyst stage embryo transfers but the difference did not reach statistical significance $(25 \%, 53.3 \%, 50 \%$; respectively $p>0.05)$. Furthermore, partial or total embryo hatching did not result in a favorable outcome compared to a control group either in women over $35(23.7 \%$, $22.9 \%, 24.6 \%)$ or younger than 35 years of age $(28.6 \%, 30.3 \%$, $25.0 \%, p>0.05$ ). In the whole study group, pregnancy rate was significantly higher in the group with blastocyst stage embryo transfers $(22.4 \%$ versus $48.8 \%, p<0.05)$

\section{Regression analysis}

A model of multivariate regression analysis was created including variables of age, serum $\mathrm{FSH}$, number of total oocytes, groups of different $\mathrm{AH}$, and the stages of transferred embryos, analysis revealed that only the stage of embryo transferred (beta coefficient $=0.175, p=0.001$ ) was shown to be significantly associated with clinical pregnancy rate after adjustment for the other variables.

\section{Discussion}

Our data analyses showed that, partial or total AH had no significant effect on the clinical implantation rate neither in cases with cleavage stage embryo transfers nor the blastocyst stage. Additionally, AH was not found to result in significant difference neither in women over 35 nor the cases $<35$ years of age. $\mathrm{AH}$ is a procedure usually applied to mimic the normal hatching stage of embryonic development, this procedure was shown to have a significant impact on the cycle outcome especially in a subset of cases with poor prognosis (i.e. at least two ART cycles, who are 38 years or older, or have poor-quality embryos). In addition to the inconsistent results regarding the favorable effect, the best technique has not yet been determined. Some methods have been suggested for AH such as mechanically, by laser energy, or chemically, by acid solution

Table 1: Summary of the demographic characteristics of the studied population

\begin{tabular}{lcc}
\hline & Mean \pm SD & $\begin{array}{c}\text { Range } \\
\text { (min-max) }\end{array}$ \\
\hline Age (Years) & & $24.0-37$ \\
FSH (mlU/mL) & $34.1 \pm 4.2$ & $4-10$ \\
Estradiol $(\mathrm{pg} / \mathrm{mL})$ & $6.8 \pm 1.7$ & $23-80$ \\
Total gonadotropin dose (units) & $47.1 \pm 15.3$ & $1000-6100$ \\
Duration of stimulation (days) & $2597.6 \pm 1168.7$ & $6-15$ \\
Number of total oocytes & $8.86 \pm 1.7$ & $5-24$ \\
Number of mature oocytes & $8.1 \pm 3.2$ & $5-20$ \\
\hline
\end{tabular}

FSH: Follicle - stimulating hormone

Table 2: Comparison of the demographic characteristics of the groups with cleavage versus blastocyst stage embryo transfers

\begin{tabular}{|c|c|c|c|}
\hline & $\begin{array}{c}\text { Cleavage (n:361) } \\
\text { versus Blastocyst }(n: 43)\end{array}$ & Mean \pm SD & ${ }^{*} p$ value \\
\hline \multirow[t]{2}{*}{ Age (Years) } & Cleavage & $34.9 \pm 4.1$ & \\
\hline & Blastocyst & $32.9 \pm 4.4$ & 0.003 \\
\hline \multirow[t]{2}{*}{$\mathrm{FSH}(\mathrm{mIU} / \mathrm{mL})$} & Cleavage & $7.01 \pm 2.05$ & \\
\hline & Blastocyst & $6.5 \pm 1.5$ & 0.134 \\
\hline \multirow[t]{2}{*}{ Estradiol (pg/mL) } & Cleavage & $47.5 \pm 15.5$ & \\
\hline & Blastocyst & $43.2 \pm 14.8$ & 0.101 \\
\hline \multirow[t]{2}{*}{ Total dose (units) } & Cleavage & $2618.2 \pm 1171.6$ & \\
\hline & Blastocyst & $2425 \pm 1143.4$ & 0.306 \\
\hline \multirow[t]{2}{*}{ Duration of stimulation (days) } & Cleavage & $8.8 \pm 1.6$ & \\
\hline & Blastocyst & $8.8 \pm 1.8$ & 0.189 \\
\hline \multirow[t]{2}{*}{ Number of total oocytes } & Cleavage & $7.8 \pm 3.1$ & \\
\hline & Blastocyst & $10.4 \pm 3.9$ & $<0.001$ \\
\hline \multirow[t]{2}{*}{ Number of mature oocytes } & Cleavage & $6.3 \pm 2.4$ & \\
\hline & Blastocyst & $8.4 \pm 3.4$ & $<0.001$ \\
\hline
\end{tabular}


Table 3: Comparison of the demographic characteristics among groups with partial, complete or no assisted hatching

\begin{tabular}{|c|c|c|c|c|c|c|}
\hline & $\mathrm{n}$ & Mean $\pm S D$ & $\begin{array}{l}\text { Range } \\
\text { (min-max) }\end{array}$ & $\begin{array}{l}\text { Lower } \\
\text { Bound }\end{array}$ & $\begin{array}{l}\text { Upper } \\
\text { Bound }\end{array}$ & $\begin{array}{c}p \\
\text { value }\end{array}$ \\
\hline \multirow[t]{4}{*}{ Age (Years) } & Partial(118) & $37.1 \pm 2.1$ & $35.0-43.0$ & 36.6 & 37.4 & \\
\hline & Total(81) & $36.5 \pm 1.8$ & $35.0-43.0$ & 36.1 & 36.9 & $<0.001$ \\
\hline & Control(205) & $32.6 \pm 4.7$ & $24.0-44.0$ & 31.9 & 33.3 & \\
\hline & Total(404) & $34.7 \pm 4.2$ & $24.0-44.0$ & 34.2 & 35.1 & \\
\hline \multirow[t]{4}{*}{$\mathrm{FSH}(\mathrm{mIU} / \mathrm{mL})$} & Partial & $7.6 \pm 2.2$ & $4-10$ & 7.2 & 8.07 & \\
\hline & Total & $6.1 \pm 2.1$ & $4-10$ & 5.7 & 6.6 & $<0.001$ \\
\hline & Control & $6.8 \pm 1.6$ & $4-10$ & 6.6 & 7.08 & \\
\hline & Total & $6.9 \pm 2.01$ & $4-10$ & 6.7 & 7.1 & \\
\hline \multirow[t]{4}{*}{ Estradiol (pg/mL) } & Partial & $49.5 \pm 16.4$ & $23-83$ & 46.5 & 52.5 & \\
\hline & Total & $41.7 \pm 14.4$ & $21-75$ & 38.5 & 44.9 & 0.001 \\
\hline & Control & $47.8 \pm 14.9$ & $10-79$ & 45.7 & 49.9 & \\
\hline & Total & $47.1 \pm 15.5$ & $10-83$ & 45.6 & 48.6 & \\
\hline \multirow[t]{4}{*}{ Total gonadotropin Dose (Units) } & Partial & $2872.5 \pm 1240.4$ & $1100-5850$ & 2646.4 & 3098.7 & \\
\hline & Total & $2512.3 \pm 1035.3$ & $1050-5850$ & 2283.4 & 2741.2 & 0.009 \\
\hline & Control & $2473.1 \pm 1154.7$ & $1000-6100$ & 2314.1 & 2632.1 & \\
\hline & Total & $2597.6 \pm 1168.7$ & $1000-6100$ & 2483.3 & 2712 & \\
\hline \multirow[t]{4}{*}{ Duration of stimulation (days) } & Partial & $8.80 \pm 1.6$ & $6-13$ & 8.5 & 9.09 & \\
\hline & Total & $9.10 \pm 1.8$ & $6-15$ & 8.6 & 9.5 & 0.054 \\
\hline & Control & $8.80 \pm 1.6$ & $6-14$ & 8.5 & 9.04 & \\
\hline & Total & $8.86 \pm 1.7$ & $6-15$ & 8.6 & 9.03 & \\
\hline \multirow[t]{4}{*}{ Number of total oocytes } & Partial & $6.7 \pm 1.1$ & $5-10$ & 6.5 & 6.9 & \\
\hline & Total & $6.8 \pm 1.1$ & $6-9$ & 6.6 & 7.1 & $<0.001$ \\
\hline & Control & $9.3 \pm 1.1$ & $6-24$ & 7.7 & 8.4 & \\
\hline & Total & $8.1 \pm 1.1$ & $6-24$ & 7.7 & 8.4 & \\
\hline \multirow[t]{4}{*}{ Number of mature oocytes } & Partial & $5.6 \pm 1.1$ & $5-9$ & 5.4 & 5.8 & \\
\hline & Total & $5.8 \pm 1.2$ & $5-9$ & 5.5 & 6.1 & $<0.001$ \\
\hline & Control & $7.4 \pm 3.3$ & $5-20$ & 6.9 & 7.9 & \\
\hline & Total & $5.38 \pm 3.5$ & $5-20$ & 5.03 & 5.73 & \\
\hline
\end{tabular}

FSH: Follicle-stimulating hormone

methods (22). Following the introduction of the best technique to be laser $\mathrm{AH}$, studies started to investigate the partial or total zona hatching on the ART outcome (23-26). Additional studies on the frozen-thawed embryo cycles revealed variable results $(27,28)$. Age also has been an issue and assessed in several further studies to determine whether there is an optimal age group, who would benefit from $\mathrm{AH}$, again these studies showed variable results (29-31). Some other variable results have been reported for the cases with recurrent implantation failure $(31,32)$. There are also studies that have been conducted to determine the impact of ZP features on reproduction, among one of these studies, ZP thickness variation was not shown to be associated with implantation after day 3 transfer and AH did not have significant impact either (33). Additionally, different rates of the size of the ZP thinning area by laser-assisted hatching were assessed in a previous study. Study compared group of embryos at cleavage stages that were not zona-manipulated, group with a quarter of the ZP was thinned using laser-assisted, half of ZP was thinned in one of the other group and in the third group, two-thirds of the ZP was thinned. Analyses of the data revealed that in vitrified-warmed embryo transfers at the cleavage-stage, the two-thirds ZP thinning group demonstrated a significantly decreased blastocyst formation rate compared with the control group, while the half ZP thinning group demonstrated a significantly increased complete hatching rate compared with the control group, authors suggested this result to have a high value in clinical application (34). Contrary to this finding, according to our data, we had cases with cleavage stage embryo transfers with partial or total $\mathrm{AH}$, data analysis showed similar clinical pregnancy rates, this result showed us that total or partial $\mathrm{AH}$ do not result in decreased blastocyst formation. Although AH was not shown to have a favorable effect in our study, in a meta-analysis of 36 randomized controlled trials with 6459 participants, AH was shown to be associated with an increased chance of achieving clinical pregnancy and multiple pregnancies. Authors pointed out the lack of data regarding whether AH significantly changes live birth and miscarriage rates (35). Due to the inconsistent results, studies have been conducted to assess efficacy of $\mathrm{AH}$ in some subgroup of women, the efficacy of laser assisted hatching of embryos on 
implantation, and pregnancy rates was assessed in a subgroup of women if they fell in either one or more of the following categories: (i) Patients over 37 years of age undergoing either IVF or ICSI treatment cycles; (ii) patients with more than 2 previous treatment cycle failures; (iii) patients undergoing frozen embryo replacement cycles, and (iv) women who were considered to be poor responders. Subjects were divided into 3 groups as: total LAH; group 1, partial LAH; group 2 and the quarter LAH; group 3. Analyses of the data showed that pregnancy and clinical pregnancy rates arising from quarter LAH were higher in comparison with partial, and total LAH (19). Another study concentrated on subgroup of women with recurrent implantation failure, in their study partial assisted hatching was shown to be superior over total hatching in terms of implantation, clinical pregnancy and live birth rates under 38 years old (36). AH was also assessed in another subgroup of women with endometriosis, authors of this study concluded that "That laserassisted hatching by thinning of the zona pellucida may be a suitable method to improve the ICSI-ET outcomes, in term of the implantation and the pregnancy rates, in cases of endometriosis" (37). Studies have been published up-to-date on the impact of $\mathrm{AH}$ with conflicting results, however among all the indications, most consistently proposed indication that the procedure had a beneficial effect was the AH in cases with repeated failures of embryo implantation. However, previous study suggested that the potential value of $\mathrm{AH}$ for indications other than repeated failure has to be weighed carefully to make sure that $\mathrm{AH}$ does not reduce the chances of implantation (38).

Additionally, in another study, $\mathrm{AH}$ was shown to result in favorable outcome especially in women with poor prognosis including those with prior failed IVF cycles (39). We conducted this study due to the paucity of data indicating the role of $\mathrm{AH}$ on the outcome in cases without known poor prognosis with their first fresh cycles (21).

To the best of our knowledge, this is the first study comparing different assisted hatching techniques on the cycle outcome on normoresponders, in our study randomization was not provided by a well-defined randomization technique, this may be accepted as a drawback in this study.

In conclusion, among the cases without known poor prognosis, and with their first fresh cycles, partial or total assisted hatching do not have any impact on the clinical pregnancy rates, no significant impact was determined in subgroup of women either.

\section{: Acknowledgements: None}

Disclosure statement: All authors declare that they have no conflict of interest.

Funding: None

\section{References}

1. Plachot M. Viability of preimplantation embryos. Baillieres Clin Obstet Gynaecol. 1992;6(2):327-38.
2. Cohen J, Alikani M, Liu HC, Rosenwaks Z. Rescue of human embryos by micromanipulation. Baillieres Clin Obstet Gynaecol. 1994;8(1):95-116.

3. Yaron Y, Botchan A, Amit A, Peyser MR, David MP, Lessing JB. Endometrial receptivity in the light of modern assisted reproductive technologies. Fertil Steril. 1994;62 (2):225-32.

4. Drobnis EZ, Andrew JB, Katz DF. Biophysical properties of the zona pellucida measured by capillary suction: is zona hardening a mechanical phenomenon? J Exp Zool. 1988;245(2):206-19.

5. Cohen J, Malter H, Fehilly C, Wright G, Elsner C, Kort H, et al. Implantation of embryos after partial opening of oocyte zonapellucida to faciliate sperm penetration. Lancet. 1988;2(8603):162.

6. Alikani M, Cohen J, Liccardi FL et al. Micromanipulation: In: Asch RH, Studd JWW (eds) Annual Progress in Reproductive Medicine. Parthenon, New York, pp. 1993:1-18.

7. al-Nuaim LA, Jenkins JM. Assisted hatching in assisted reproduction. BJOG. 2002;109(8):856-62.

8. Magli MC, Gianaroli L, Ferranetti AP, Fortini D, Aicardi G, Montanaro N. Rescue of implantation potential in embryos with poor prognosis by assisted zona hatching. Hum Reprod. 1998;13(5):1331-5.

9. Primi MP, Senn A, Montag M, Van der Ven H, Mandelbaum J, Veiga A, et al. A European multicentre prospective randomized study to assess the use of assisted hatching with a diode laser and the benefit of an immunosuppressive/antibiotic treatment in different patient populations. Hum Reprod. 2004;19(10):2325-33.

10. Gabrielsen A, Agerholm I, Toft B, Hald F, Petersen K, Aagaard J, et al. Assisted hatching improves implantation rates on cryopreserved-thawed embryos. A randomized prospective study. Hum Reprod. 2004;19(10):2258-62.

11. Edi-Osagie E, Hooper L. Seif MW. The impact of assisted hatching on live birth rates and outcomes of assisted conception: a systematic review. Hum Reprod. 2003;18(9): 1828-35.

12. Sallam HN, Sadek SS, Agameya AF. Assisted hatching a meta-analysis of randomized controlled trials. J Assist Reprod Genet. 2003;20(8):332-42.

13. Tao J, Tamis R. Application of assisted hatching for 2-dayold, frozen-thawed embryo transfer in a poor-prognosis population. J Assist Reprod Genet. 1997;14(2):128-30.

14. Chao Kh, Chen SU, Chen HF, Wu MY, Yang YS, Ho HN. Assisted hatching increases the implantation and pregnancy rate of in vitro fertilization (IVF)-embryo transfer (ET), but not that of IVF-tubal ET in patients with repeated failures. Fertil Steril. 1997;67(5):904-8.

15. Isik AZ, Vicdan K, Kaba A, Dagli G. Comparison of zona manipulated and zona intact blastocyst transfer: a prospective randomised trial. J Assist Reprod Genet. 2000; 
17(3):135-9.

16. Nakayama T, Fujiwara H, Yamada S, Tastumi K, Honda T, Fujii S. Clinical application of anew assisted hatching method using a piezo-micromanipulator for morphologically low-quality embryos in poor prognosis infertile patients. Fertil Steril. 1999;71(6):1014-18.

17. Germond M, Primi MP, Senn A et al. The use of laser for micromanipulation. In: Shoham Z, Howles CM, Jacobs S (eds) Female Infertility Therapy Current Practice. Martin Dunitz, London, pp. 1999:221-232.

18. Germond M, Nocera D, Senn A, Rink K, Delacrétaz G, Fakan S. Microdissection of mouse and human zonapellucida using a $1.48 \mu \mathrm{m}$ diode laser beam: efficacy and safety of the procedure. Fertil Steril. 1995;64(3):604-11.

19. Mantoudis E, Podsiadly BT, Gorgy A, Venkat G, Craft IL. A comparison between quarter, partial and total laser assisted hatching in selected infertility patients. Hum Reprod. 2001;16(10):2182-6.

20. Hendawy SF, Raafat T. Comparison between Cleavage Stage versus Blastocyst Stage Embryo Transfer in an Egyptian Cohort Undergoing in vitro Fertilization: A Possible Role for Laser Assisted Hatching. Clin Med Insights Reprod Health. 2011;5:41-8.

21. Carney SK, Das S, Blake D, Farquhar C, Seif MM, Nelson L. Assisted hatching on assisted conception (in vitro fertilisation (IVF) and intracytoplasmic sperm injection (ICSI). Cochrane Database Syst Rev. 2012;12:CD001894.

22. Quaas A, Dokras A. Diagnosis and treatment of unexplained infertility. Rev Obstet Gynecol. 2008;1(2): 69-76.

23. Moraloglu O, Kilic S, Karayalcin R, Yuksel B, Tasdemir $\mathrm{N}$, Isik A, et al. Comparison of GnRH agonists and antagonists in normoresponder IVF/ICSI in Turkish female patients. Adv Ther. 2008;25(3):266-73.

24. Lainas T, Zorzovilis J, Petsas G, Stavropoulou G, Cazlaris H, Daskalaki V, et al. In a flexible antagonist protocol, earlier, criteria-based initiation of GnRH antagonist is associated with increased pregnancy rates in IVF. Hum Reprod 2005;20(9):2426-33.

25. Ghobara TS, Cahill DJ, Ford WC, Collyer HM, Wilson PE, Al-Nuaim L, et al. Effects of assisted hatching method and age on implantation rates of IVF and ICSI. Reprod Biomed Online. 2006;13(2):261-7.

26. Padula F, Capriglione S, Iaconianni P, Gatti S, Lippa A, Minutolo SE, et al. Laser-assisted hatching of human embryos: may two alternative approaches (thinning versus drilling) impact on implant rate? Lasers Med Sci. 2017;32(7):1663-6.

27. González-Ortega C, Cancino-Villarreall P, Anaya-Torres FJ, Pérez-Peña E, Gutiérrez-Gutiérrez AM. Impact of laser-assisted hatching (quarter technique) in poor prognosis Patients. Ginecol Obstet Mex. 2015;83(11):670-9.

28. Ren X, Liu Q, Chen W, Zhu G, Zhang H. Effect of the site of assisted hatching on vitrified-warmed blastocyst transfer cycles: a prospective randomized study. J Assist Reprod Genet. 2013;30(5):691-7.

29. Debrock S, Peeraer K, Spiessens C, Willemen D, De Loecker P, D'Hooghe TM. The effect of modified quarter laser-assisted zona thinning on the implantation rate per embryo in frozen/vitrified-thawed/warmed embryo transfer cycles: a prospective randomized controlled trial. Hum Reprod. 2011;26(8):1997-2007.

30. Hiraoka K, Hiraoka K, Horiuchi T, Kusuda T, Okano S, Kinutani $\mathrm{M}$, et al. Impact of the size of zona pellucida thinning area on vitrified-warmed cleavage-stage embryo transfers: a prospective, randomized study. J Assist Reprod Genet. 2009;26(9-10):515-21.

31. Balaban B, Urman B, Yakin K, Isiklar A. Laser-assisted hatching increases pregnancy and implantation rates in cryopreserved embryos that were allowed to cleave in vitro after thawing: a prospective randomized study. Hum Reprod. 2006;21(8):2136-40.

32. Ng EH, Naveed F, Lau EY, Yeung WS, Chan CC, Tang OS, et al. A randomized double-blind controlled study of the efficacy of laser-assisted hatching on implantation and pregnancy rates of frozen-thawed embryo transfer at the cleavage stage. Hum Reprod. 2005;20(4):979-85.

33. Kutlu P, Atvar O, Vanlioglu OF. Laser assisted zona thinning technique has no beneficial effect on the ART outcomes of two different maternal age groups. J Assist Reprod Genet. 2010;27(8):457-61.

34. Hagemann AR, Lanzendorf SE, Jungheim ES, Chang AS, Ratts VS, Odem RR. A prospective, randomized, doubleblinded study of assisted hatching in women younger than 38 years undergoing in vitro fertilization. Fertil Steril. 2010;93(2):586-91.

35. Valojerdi MR, Eftekhari-Yazdi P, Karimian L, Ashtiani SK. Effect of laser zona pellucida opening on clinical outcome of assisted reproduction technology in patients with advanced female age, recurrent implantation failure, or frozen-thawed embryos. Fertil Steril. 2008;90(1):84-91.

36. Rufas-Sapir O, Stein A, Orvieto R, Avrech OM, Kotler N, Pinkas $\mathrm{H}$, et al. Is assisted hatching beneficial in patients with recurrent implantation failures? Clin Exp Obstet Gynecol. 2004;31(2):110-2.

37. Nada AM, El-Noury A, Al-Inany H, Bibars M, Taha T, Salama S, et al. Effect of laser-assisted zona thinning, during assisted reproduction, on pregnancy outcome in women with endometriosis: randomized controlled trial. Arch Gynecol Obstet. 2018;297(2):521-28.

38. Germond M, Primi MP, Senn A. Hatching: how to select the clinical indications. Ann N Y Acad Sci. 2004;1034: 145-51.

39. Pfeifer S, Lobo R, Sokol R, Goldberg J, Fossum G, Thomas M, et al. Role of assisted hatching in in vitro fertilization: a guideline. Fertil Steril. 2014;102(2):348-51. 